\title{
The Improving Water Shortage Problem Intervention Plan For Egypt
}

\author{
Huan Meng \\ School of North China Electric Power University, Baoding 071000, China; \\ 619537030@qq.com
}

Keywords: Intervention Plan, Dynamic Recommendation Model

\begin{abstract}
In this paper, some intervention plans are established to find proper ways to improve water shortage problem for Egypt.Also,it is known to all that a intervention plan will lose its effects and validity one day. Due to this reason, a dynamic recommendation model based on Markov chain is adopted to optimize the intervention plan. It can decide whether the plan is worthwhile carrying out by calculating the availability of water.
\end{abstract}

\section{Introduction}

Water is essential for life. One quarter of the world's population experience water scarcity due to the limited amount of fresh water. Physical scarcity and economic scarcity are considered as two primary causes of water scarcity. Stepping in the modern society, requirement of water resources for residential and agricultural are rapidly increasing as a result of the growing global population. At the same time, industrial consumption and pollution also bring a heavy burden on the supply of fresh water. In order to handle the existing water crisis, scientists have proposed several ways to get clean water or industrial water-desalination of sea water, sewerage system, and rainwater harvesting techniques. Meanwhile more water saving techniques are on their way to be popularized.

\section{Water supply augmentation plan}

Judging from Figure 1 and Figure 2 ,we can get the fact that,these limited quantities of water have to fulfill the Egyptian requirements in the fields of agriculture, which is the largest consumer of water (85\%), industry and domestic uses. Each resource has its usage limitation, whether these limitations are related to quantity, quality, space, time, or exploitation cost. To solve this problem, we recommend two ways to increase water supply in Egypt.

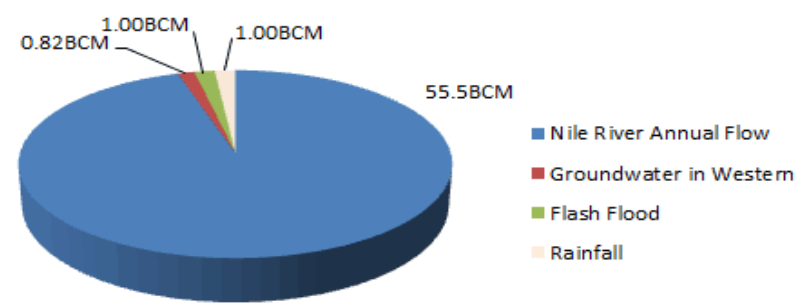

Figure 1: water resource distribution

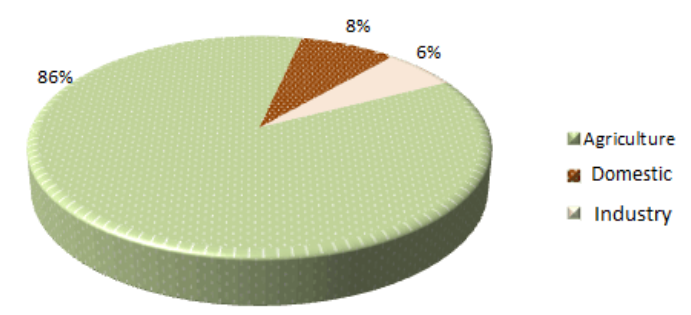

Figure 2: water use allocation in Egypt

\section{Reuse of drainage water and treated wastewater}

Egypt is one of the pioneer countries in the reuse of water. We came up with the plan of building wastewater treat plants. And the possible following plan can be using this water for the cultivation of special crops (timber trees, industrial crops such as cotton, flax, flowers, etc.). 


\section{Desalination of brackish and sea water}

Desalination has long been confined to situations where no other alternatives were available to produce drinking water. We hold the view that desalination should be taken as a serious option for the production of drinking and industrial water as an alternative to traditional surface water treatment in Egypt.There is unlimited potential for further development of seawater desalination in Egypt along the long shoreline(in the Mediter- ranean Region resource and Red Sea coastal area). And considering the vast reserves of brackish groundwater in Egypt, there is also great potential for brackish water desalination which can be applied at much lower cost.

\section{Water saving plan}

Reducing water consumption is the priority in tackling the water crisis in Egypt. Water consumption in agriculture is $85 \%$ of the total consumption means that,we should bring the agricultural water requirements to a minimum in order to make sufficient quantities available for other activities that might be of higher priority (such as drinking water supply) or of higher return per unit volume of water (such as industry or tourism) ${ }^{[1]}$.

Using modern irrigation systems and changing surface irrigation to drip irrigation

It is obligatory to use sprinkler and drip irrigation systems in the desert lands converted into agricultural production.

\section{Modification of the cropping pattern}

Shifting to less water-demanding crops. For instance, introducing cropping patterns with low water requirements ${ }^{[1]}$ (i.e. abandoning rice and sugarcanes for other cropping patterns).

\section{Birth control}

Advancements in living standards together with population growth have already been reflected in expansion of water consumption levels for domestic use. Domestic water use grew from 3.1 BCM in 1990 (Abu-Zeid,1991) to 5.23 BCM in 2000 (FAO Aqua- stat). Further augmentation of the life quality and the population growth will push up water demands ${ }^{[2]}$. From this point of fact,we recommend a birth control of Egypt in the intervention plan.

\section{Education and Awareness}

Finally,introducing public awareness campaigns aimed towards advocating a new water culture in a society based on the principle of conservation. The significance of water conservation in irrigation and domestic uses can be promoted through such public awareness campaigns.

Thus, the relationship between all kinds of inventions are described as follows, including economy, agriculture, politics ,Industry etc. 


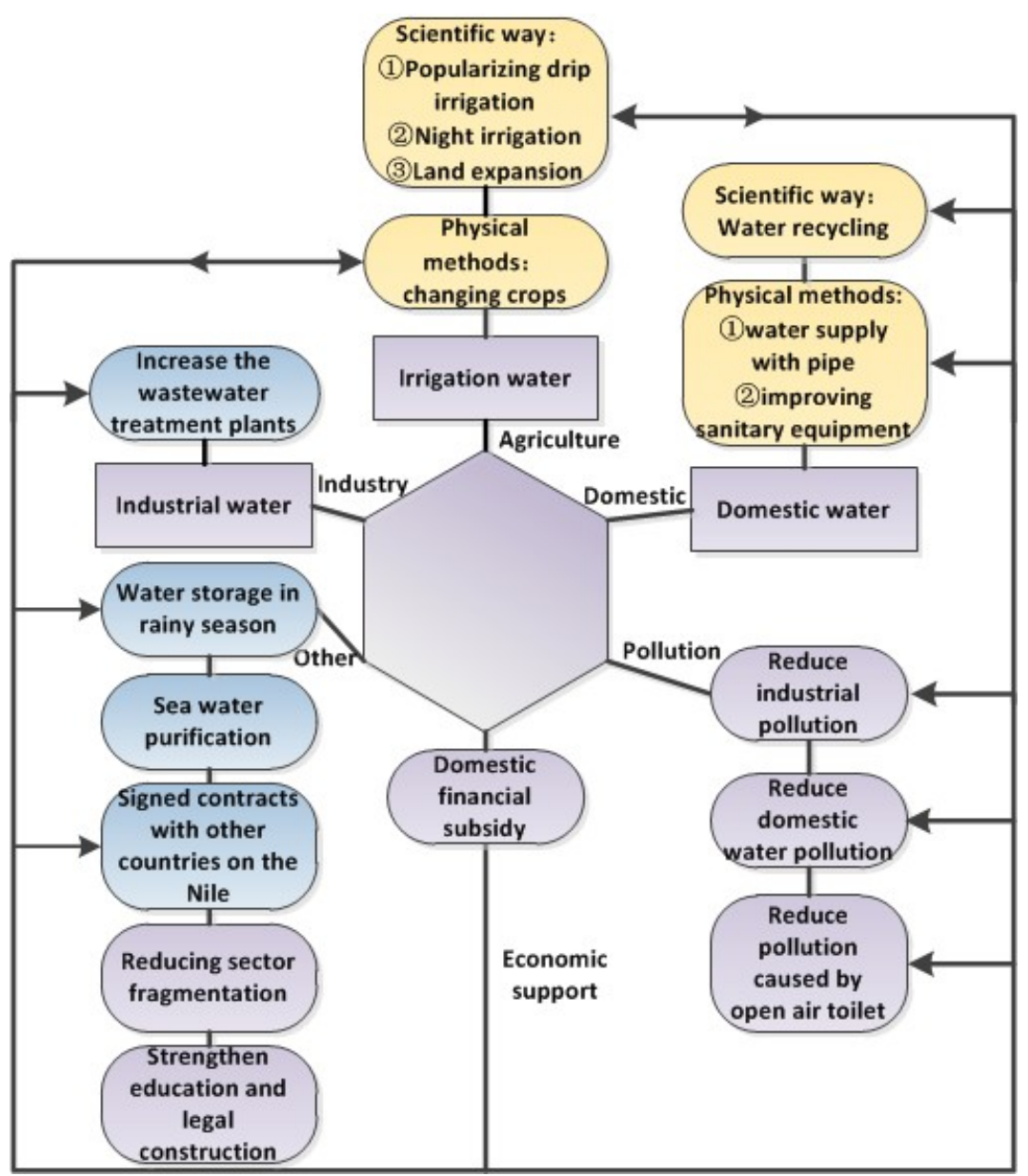

Figure 3: Relationship between all kinds of interventions

\section{Optimization of intervention plan}

\section{The Dynamic Recommendation Model of Markov Chain}

In this part,we see the above discussed problem as an optimization problem and set water availability $F w$ as an objective function. The implementation of Intervention program will change the weights of all the indexes in the following way: $w_{i}=k_{i} w_{i 0}$.A number of sub programs contained in the intervention plan can be described as a collection of $P=\{P 1, P 2, P 3, \ldots, P m\}$, the implementation time of $P i$ is $t i$. The intervention plan will be carried on,in condition that $F w$ is optimized after the application of plan $P i$.

Otherwise,the program will be terminated. In order to simplify the model,we assume that each sub plan is able to be adopted at the same time ${ }^{[3]}$.

Step1. Obtaining Data

The obtained data include a series of indicators reflecting the availability of water resources in a country or region,marked as a set of indicators $T a=\{T a 1, T a 2, T a 3, \ldots, T$ an $\}, \Delta n$ indicators are possible to be either increased or reduced in a time period of $\Delta \tau$.

In this model,we assume that the index is not changed.In addition,the database needs noise removal treatment, and updating in an interval of $\Delta \tau$. If the data has deviation,it will have an impact on the objective function, shown as the following equation:the delta $\mathrm{Fw}+\Delta \varepsilon$.

Step2. Adopting Association Rules Recommendation Algorithm ${ }^{[3]}$ Calculated the correlation matrix $C$ between each index ,

$$
C=\left(\begin{array}{ccc}
C_{11} & \ldots & C_{1 n} \\
\vdots & \ddots & \vdots \\
C_{n 1} & \cdots & C_{n n}
\end{array}\right) \text { (Suppose including } n \text { indexs) }
$$


If the weight of index $i$ is changed by the intervention plan,according to the recommendation algorithm, one or several indicators closely associated with the index will be recommended.We can also use the concept of Markov chain ,to explore those less closely related indexes of $i$ replace the weight of nonrelated index according to probability $p$, and change the weight of related indexes by using probability $(1-p)$.

Step3. Inspection of Step2

After updating index weights in step2, we need to recalculate Fw after a period time of $\Delta \tau$.If $F w$ is optimized,continue the execution of correction plan in Step2.Otherwise,return to the original intervention plan.

\section{Advantages of the Model}

The process mentioned above dynamically changes intervention program ,namely index weight through a time interval of $\Delta \tau$. The model also continues seeking optimization of the objective function.It has a high robustness. What is more, the water availability can also be predicted through this model by using original data.

\section{Shortcomings of the Model}

This model has a complicated calculation process, which can be tackled by using mathematical optimization iteration.

\section{References}

[1]Abdin A E,Gaafar I. Rational water use in Egypt[J]. M. El Moujabber, L. Mandi, G. Trisorio Liuzzi, I. Martin, A. Rabi, R. Rodriguez (eds), 2009: 11-28.

[2]MOHAMED ELSHOPKY, MAJOR., EGYPTM.Sc., Commanders and Staff Officers College, Cairo, 2008 B.Sc., Military Academy, Cairo, 1998 THE IMPACT OF WATER SCARCITY ON EGYPTIAN NATIONAL SECURITY AND ON REGIONAL SECU- RITY IN THE NILE RIVER BASIN,2012-01

[3]http://blog.jobbole.com/72373/ 